\title{
Basic Equation for the Different Coupled Equations between the First and Second Laws of Thermodynamics at Strong Coupling
}

\author{
Saeed Shahsavari \\ ${ }^{1}$ Department of Mechanical Engineering, Isfahan University of Technology \\ Isfahan, Iran. \\ Email: s.shahsavari [AT] me.iut.ac.ir
}

\begin{abstract}
For some physical processes, the first and second laws of thermodynamics can be at strong coupling. Also, it is possible that the familiar inequalities of macroscopic thermodynamics cannot be used in the analysis of the system, and it is needed that the inequalities rewrote as equalities. In these cases, the work, internal energy, dissipated energy, and entropy production must be considered and identified together. In this paper, the basic equation for the different coupled equations between the first and second laws of thermodynamics at strong coupling is extracted. Also, inspired by the first and second laws of thermodynamics and different approaches to the second law, a thermophysical equation for thermodynamics is extracted. This equation can be used instead of the first and second laws of thermodynamics as to the analysis of the performed process these laws must be established together. It is tried that effective internal energy, directly to be related to the entropy or vice versa, in one general equation. Also, the presented equation is in the same line with the different approaches to the second law and energy structure theory.
\end{abstract}

Keywords--- First law of thermodynamics; Second law of the thermodynamics; Strong coupling; Internal energy; Entropy production; Thermophysical equation; Energy structure theory

\section{INTRODUCTION}

Two mains of the physical laws are the first and second laws of thermodynamics [1]. While the second law of thermodynamics is introduced by presenting Carnot's cycles, it can be understood without any assumption about such as irreversibility, heat or temperature, etc [2]. And also, it can be derived by using some physical theories [3]. To investigate some general physical processes, the general second law of thermodynamics is extracted [4-6]. Also, the second law of thermodynamics in statistical physics is investigated to the quantum regime [7]. The second law of thermodynamics is used in electromagnetic theory [8]. One important question is about the second law if time is stopping [9]. Also, second law of thermodynamics can be used to design in the physical processes [10-11]. In some physical processes, the first and second laws are at a strong coupling that it is needed that these laws apply to the system together. Also, that may be needed that the inequalities be rewritten as equalities. The classical, statistical, quantum and Kinetic theory approaches of the second law can be used for these purposes [12-16]. While there are some challenges to the second law of thermodynamics [17].

Energy structure theory is presented to investigate the effects of the second law of thermodynamics on the physical processes from the perspective of the independent and dependent energy components of the system [18,19]. This theory studies the structure of the irreversibility and takes the reasons and conditions of the entropy production in feasible processes. In other words, energy structure theory extract a macroscopic quasi-statistical equation as the energy structure equation of the system, and by studying its variation in different paths, a new approach to the second law and irreversibility concept is presented. Therefore, this theory can be a suitable idea to investigate the first and second laws of thermodynamics at strong coupling.

In this paper, the basic equation for the different coupled equations between the first and second laws of thermodynamics at strong coupling is studied. Also, inspired the first and second laws of thermodynamics as well as different approaches to the second law of thermodynamics, and also using the energy structure theory concepts, a thermophysical equation is extracted for the thermodynamics as the first and second laws are in the strong coupling.

\section{FIRST AND SECOND LAWS OF THERMODYNAMICS AT STRONG COUPLING}

Equations (1) and (2) present the first and second law of thermodynamics for a Thermodynamic cycle [1]:

$\oint \delta Q=\oint \delta W$ 
$\oint \frac{\delta Q}{T} \leq 0$

Where $\delta W$ is the work done by system, $\delta Q$ is the heat exchange and $T$ is temperature. The first law explains that energy is conserved, and the second law presents a condition relation to the thermodynamic cycles. Also, the second law introduces the irreversibility concept in physical processes. These laws can be presented as following in variation form [1]:

$\delta U=\delta Q-\delta W$

$d s \geq \frac{\delta Q}{T}$

That $U$ is the internal energy and $s$ is entropy.

While equation (3) does not any attitude to the entropy, equation (4) uses entropy as a basic quantity but does not take any value to the entropy variation and only presents a condition related to it. Equation (4), directly does not any attitude to the work done, while the work done can cause the generated entropy. Therefore, entropy will do not dependent on the total value of the internal energy, as depends on a part of internal energy. This part of internal energy can be considered as the effective internal energy. From the perspective of the energy structure theory, the effective energy depends on how energy is applied to the system as well as its amount [18]. And also remaining depends on the amount of applied energy.

To investigate a physical system at strong coupling of the first and second law of thermodynamics, not only work but also dissipated energy, internal energy, and generated entropy must be identified on the level of a trajectory of the system [12]. From the classical point of view and by using the total Hamiltonian trajectory, equation (5) can be derived:

$<\exp \left[-\Delta s_{t o t}\left(\epsilon^{0}\right)\right]>=1$

Where $\Delta s_{t o t}$ is the total entropy change and $\epsilon^{0}$ is the initial point phase.

Also statistically, the forward and reverse processes can be investigated. If the forward and reverse processes are described by work distributions $\rho_{F}(W)$ and $\rho_{R}(W)$, equation (6) can be derived [13]:

$\frac{\rho_{F}(+W)}{\rho_{F}(-W)}=\exp \left[\frac{<W-\Delta F\rangle}{k_{B} T}\right]$

And also:

$<\exp \left(\frac{-W}{k_{B} T}\right)>=1$

Where $F=U-T S$ and $k_{B}$ is the Boltzmann constant. Also, equation (8) is presented as a time-dependent weight by using Poisson bracket notation $[13,20]$ :

$\frac{\partial g}{\partial t}+\{g, H\}=-\frac{\dot{W}}{k_{B} T} g$

Where $\dot{W}=\dot{\lambda} \frac{\partial H}{\partial \lambda}$ that $\lambda$ is the work parameter and $g$ is the weighted density. And also, from the perspective of quantum mechanics, some operators are suggested as internal energy operators [12].

\section{ENTROPY PRODUCTION FROM THE PERSPECTIVE OF THE ENERGY STRUCTURE THEORY}

Entropy production is one of the most important quantities that must be considered for studying the first and second laws of thermodynamics at strong coupling. From the perspective of the energy structure theory, equation (9) can be considered as the energy structure equation of the system $[18,19]$ :

$U_{T}=\left(u_{1}+u_{2}+\cdots+u_{m}\right)+\left[g_{1}+\cdots+g_{k}\right]+\left[h_{1}+\cdots+h_{n}\right]+U_{T_{0}}$

Where:

$g_{j}=g_{j}\left(u_{1}, u_{2}, \ldots, u_{m}\right)$

$h_{p}=h_{p}\left(\dot{u}_{1}, \ldots, \dot{u}_{m}\right)$

That $u_{i}$ is an energy component.

Also, using the energy structure theory, entropy production can be extracted as follows: 
$\varphi=\sum_{i=1}^{m} \frac{\delta H_{i}}{\delta U_{T}}$

Where $\delta U_{T}$ is the applied energy to the system. And also:

$\delta H_{i}=\left(\sum_{p=1}^{n}\left(\frac{\partial h_{p}}{\partial \dot{U}_{i}}\right)\right) \delta \dot{U}_{i}$

Equation (12) explains the structure of the entropy production using energy structure components of the system as well as independent and dependent energy components concept [18].

\section{A THERMOPHYSICAL EQUATION AS THE BASIC OF THE COUPLED EQUATIONS}

Equations (3-8) present the first and second laws of thermodynamics and explain different approaches to the second law as well as the irreversibility concept. These equations, also explain the entropy production effects on the establishing of the first law as there is a strong coupling between the first and second laws in the performed process. From these equations, it can be concluded that an equation as a coupled equation between first and second laws, must have four quantities: Entropy; Applied energy; Effective internal energy; Macro state of the system.

Effective internal energy is part of the internal energy that directly, depends on the entropy. And also, applied energy is not in the form of work or heat only, rather it can be a combination of them.

Inspired by the first and second laws of thermodynamics as well as different approaches to the second law (Equations 3-8) and energy structure theory concepts, equation (14) can be resulted as a coupled equation for thermodynamics:

$\frac{\partial^{2} U_{\text {in }}^{\text {eff }}}{\partial S^{2}}=\omega\left(S, \frac{\partial U_{\text {in }}^{\text {eff }}}{\partial S}\right) \dot{U}_{a p p}$

Where $U_{a p p}$ is the applied energy to the system, $U_{\text {in }}$ is the internal energy, $U_{\text {in }}^{\text {eff }}$ is the effective internal energy, $S$ is entropy, and $\omega=\omega\left(S, \frac{\partial U_{i n}^{e f f}}{\partial S}\right)$ is a function that depends on the macro state of the system as well as how energy is applied to the system. And also, $\dot{U}_{a p p}=\frac{\partial U_{a p p}}{\partial t}$.

The general form of the equation (14) is in the same line with the equations (3-8) as well as energy structure theory. Equation (14), also takes a new approach on the basis of the different coupled equations between the first and second laws of thermodynamics at strong coupling.

If the applied energy is divided into work and heat, equation (14) can be rewritten as follows:

$\frac{\partial^{2} U_{i n}^{e f f}}{\partial S^{2}}=\omega_{W}\left(S, \frac{\partial W}{\partial S}\right) \dot{W}+\omega_{Q}\left(S, \frac{\partial Q}{\partial S}\right) \dot{Q}$

Where $\omega_{W}\left(S, \frac{\partial W}{\partial S}\right)$ depends on the work done as well as the macrostate of the system, and $\omega_{Q}\left(S, \frac{\partial Q}{\partial S}\right)$ depends on the heat as well as the macrostate of the system.

\section{CONCLUSIONS}

In some physical processes, the first and second laws of thermodynamics can be at strong coupling. To investigate these processes, four quantities must be identified: Work; Entropy production; Internal energy; Dissipated energy.

Equation (14) presents a thermophysical equation that can be used, whenever there is thermodynamically strong coupling in the performed processes. Also, equation (14) is extracted using the thermodynamically and physical properties of the system and has the same base as the statistical concepts in modern physics as well as energy structure theory.

Equation (14) has the same base as the different formulations of the second law of thermodynamics and energy structure theory concepts. This equation, also explains the bases of the different coupled equations between the first and second laws of thermodynamics at strong coupling. In fact, equation (14) can be used as the basis of the coupled equations.

Acknowledgments- While equation (14) establishes the general needed conditions to a coupled equation, but I do not have any idea to calculate the function $\omega$ now. 


\section{REFERENCE}

[1] Carnot, Sadi, Rudolf Clausius, and William Thomson Baron Kelvin. The Second Law of Thermodynamics. American Book Company, 1899.

[2] Lieb, Elliott H., and Jakob Yngvason. "The mathematical structure of the second law of thermodynamics." arXiv preprint math-ph/0204007 (2002).

[3] Gemmer, Jochen, Alexander Otte, and Günter Mahler. "Quantum approach to a derivation of the second law of thermodynamics." Physical Review Letters 86.10 (2001): 1927.

[4] Setare, M. R. "Generalized second law of thermodynamics in quintom dominated universe." Physics Letters B 641.2 (2006): 130-133.

[5] Zhou, Jia, et al. "The generalized second law of thermodynamics in the accelerating universe." Physics Letters B 652.23 (2007): 86-91.

[6] Karami, K., and A. Abdolmaleki. "Generalized second law of thermodynamics in f (T) gravity." Journal of Cosmology and Astroparticle Physics 2012.04 (2012): 007.

[7] Abe, Sumiyoshi, and A. K. Rajagopal. "Validity of the second law in nonextensive quantum thermodynamics." Physical review letters 91.12 (2003): 120601.

[8] Green, A. E., and P. M. Naghdi. "Aspects of the second law of thermodynamics in the presence of electromagnetic effects." The Quarterly Journal of Mechanics and Applied Mathematics 37.2 (1984): 179-193.

[9] Neri, Izaak. "Second law of thermodynamics at stopping times." Physical Review Letters 124.4 (2020): 040601.

[10] Sama, D. A. "The use of the second law of thermodynamics in process design." (1995): 179-185.

[11] Wilming, Henrik, Rodrigo Gallego, and Jens Eisert. "Second law of thermodynamics under control restrictions." Physical Review E 93.4 (2016): 042126.

[12] Seifert, Udo. "First and second law of thermodynamics at strong coupling." Physical review letters 116.2 (2016): 020601.

[13] Jarzynski, Christopher. "Equalities and inequalities: Irreversibility and the second law of thermodynamics at the nanoscale." Annu. Rev. Condens. Matter Phys. 2.1 (2011): 329-351.

[14] Coveney, Peter V. "The second law of thermodynamics: entropy, irreversibility and dynamics." Nature 333.6172 (1988): 409-415.

[15] Campisi, Michele. "Statistical mechanical proof of the second law of thermodynamics based on volume entropy." Studies in History and Philosophy of Science Part B: Studies in History and Philosophy of Modern Physics 39.1 (2008): 181-194.

[16] Eu, Byung C. "Kinetic theory and irreversible thersmodynamics." STIA 93 (1992): 24498.

[17] Cápek, Vladislav, and Daniel P. Sheehan. Challenges to the second law of thermodynamics. Dordrecht: Springer, 2005.

[18] Shahsavari, Saeed, Mehran Moradi, "A Study of the Entropy Production in Physical Processes from a New Perspective of the Energy Structure." Asian Journal of Applied Sciences (ISSN: 2321-0893) 8.6 (2020).

[19] Shahsavari, Saeed, Mehran Moradi, and Morteza Esmaeilpour. "On the Irreversibility in Mechanical Systems Using a New Macroscopic Energy Structure Modeling." Asian Journal of Applied Sciences (ISSN: 2321-0893) 8.6 (2020).

[20] Goldstein H. 1980. Classical Mechanics. Reading, MA: Addison-Wesley. 2nd 